\title{
How to probe a fractionally charged quasihole?
}

\author{
C. Schüller ${ }^{\mathrm{a}, *}$, K.-B. Broocks ${ }^{\mathrm{a}}$, P. Schröter ${ }^{\mathrm{a}}$, Ch. Heyn ${ }^{\mathrm{a}}$, D. Heitmann ${ }^{\mathrm{a}}$, M. Bichler ${ }^{\mathrm{b}}$, \\ W. Wegscheider ${ }^{\mathrm{c}}$, T. Chakraborty ${ }^{\mathrm{d}}$, V.M. Apalkov ${ }^{\mathrm{e}}$ \\ anstitut für Angewandte Physik und Zentrum für Mikrostrukturforschung, Universität Hamburg, Jungiusstraße 11, \\ Hamburg D-20355, Germany \\ ${ }^{\mathrm{b}}$ Walter-Schottky-Institut der TU München, Am Coulombwall, Garching D-85748, Germany \\ ${ }^{\mathrm{c}}$ Institut für Experimentelle und Angewandte Physik, Universität Regensburg, Regensburg D-93040, Germany \\ ${ }^{\mathrm{d}}$ Canada Research Chair in Physics, Department of Physics and Astronomy, \\ 223 Allen Building, University of Manitoba, Winnipeg, Manitoba, Canada R3T 2N2 \\ e Physics Department, University of Utah, Salt Lake City, UT 84112-0830, USA
}

\begin{abstract}
We report an anomalous dispersion of charged excitons in photoluminescence experiments on a two-dimensional electron gas subjected to a quantizing magnetic field around $v=\frac{1}{3}$. We have found that the anomaly exists only at a very low temperature $(0.1 \mathrm{~K})$ and an intermediate electron density $\left(0.9 \times 10^{11} \mathrm{~cm}^{-2}\right)$. It is explained to occur due to the perturbation of the incompressible liquid at $v=\frac{1}{3}$. The perturbation is induced by the close proximity of a localized charged exciton which creates a fractionally charged quasihole in the liquid. The intriguing experimentally observed puzzle that the anomaly can be destroyed by applying a small thermal energy of $\sim 0.2 \mathrm{meV}$ is thereby resolved, as this energy is enough to close the quasihole energy gap. This work presents a probe of the quasihole gap in a quantum Hall system.
\end{abstract}

(C) 2003 Elsevier B.V. All rights reserved.

PACS: 71.30.+h; 73.43.-f; 73.63.Kv; 73.43.Lp

Keywords: Quantum Hall effect; Photoluminescence; Fractionally charged particles

Fractionally charged quasiholes are the low-lying charged excitations of the incompressible liquid state proposed by Laughlin [1-3]. In addition to the ground state energy, the quasihole creation energy for the state proposed by Laughlin is also known very accurately [4]. The corresponding energy gap has been investigated experimentally (see for e.g. Ref. [5]) and theoretically $[3,6]$, and, several groups reported the detection of fractionally charged objects in the edge states of a two-dimensional electron system invoking a chiral Luttinger liquid theory [7]. In this manuscript

\footnotetext{
* Corresponding author.

E-mail address: schueller@physnet.uni-hamburg.de (C. Schüller).
}

we show that the lowest-energy charged excitations, the quasiholes, can been probed at $v=\frac{1}{3}$ (the Laughlin state) via photoluminescence (PL) spectroscopy of charged excitons.

So far, PL experiments have been intensively used to explore the highly correlated fractional quantum Hall effect (FQHE) states (see e.g., Ref. [8]). The presence of photoexcited holes is one of the striking differences as compared to electron transport experiments. This effect has been systematically studied in recent years in particular in experiments on very dilute two-dimensional electron systems (2DESs) in the density range $\sim 10^{10} \mathrm{~cm}^{-2}$. Here the ground state is formed by negatively charged excitons, a bound state of two electrons and one hole. In a magnetic field, the 
two electrons can form either a singlet or a triplet state, the excitons are then called singlet $\left(\mathrm{X}_{\mathrm{s}}^{-}\right)$or triplet $\left(\mathrm{X}_{\mathrm{t}}^{-}\right)$excitons, respectively [9].

For samples with significant disorder and at low density the 2DES breaks up into areas with finite density (electron puddles) and completely depleted regions. In the completely depleted regions, neutral excitons $\left(\mathrm{X}^{0}\right)$ can be excited. The effect of the disorder potential is one of the crucial points in our experiments. In experiments at very low temperatures and in this density regime of a 2DES, so-called dark triplet excitons were observed [10,11], which at high magnetic fields exhibit a crossing with the $X_{\mathrm{s}}^{-}$[12]. At higher densities $\left(\sim 10^{11} \mathrm{~cm}^{-2}\right)$ and for very high electron mobility, the Coulomb interaction between electrons and holes is mostly screened. In this regime, PL experiments have revealed a variety of interaction effects in the FQHE states [8]. At the crossover between these two regimes, interesting interaction related effects can be expected. Very recently, we reported the observation of a strong energetic anomaly of the charged excitons in samples with moderate electron mobility $\left(\mu \sim 10^{5} \mathrm{~cm}^{2} / \mathrm{Vs}\right)$ in, what we call an intermediate density range of about $1 \times 10^{11} \mathrm{~cm}^{-2}$ [13]. Here we show that in this special parameter range a uniform 2DES, which at filling factor $v=\frac{1}{3}$ can form a highly correlated incompressible liquid, and charged excitons coexist. We propose that perturbation of that liquid by a localized charged exciton leads to the creation of a fractionally charged quasihole in the liquid which can account for the observed anomaly.

The investigated samples are one-sided doped $25 \mathrm{~nm}$-wide GaAs- $\mathrm{Al}_{0.3} \mathrm{Ga}_{0.7}$ As single quantum wells with carrier densities around $2 \times 10^{11} \mathrm{~cm}^{-2}$ under illumination. Via external gates the carrier density can be tuned down to the range $\sim 10^{10} \mathrm{~cm}^{-2}$. Experiments are performed via glass fibers in a dilution cryostat at temperatures between $T=0.1$ and $1.8 \mathrm{~K}$ resolving circularly polarized light $[11,13]$.

Fig. 1 displays series of PL spectra at low temperature $(T=0.1 \mathrm{~K})$ for left- $(\sigma-$, Fig. $1(\mathrm{a}))$ and right-circular $(\sigma+$, Fig. 1(b)) polarizations. For $v>2$, i.e., if the lowest Landau level is completely occupied, we find a nearly linear dispersion where Landau level transitions between electrons and holes can be identified. This shows that in this range of filling factors the system behaves like a uniform 2DES where excitonic effects are not well pronounced. On the other hand,

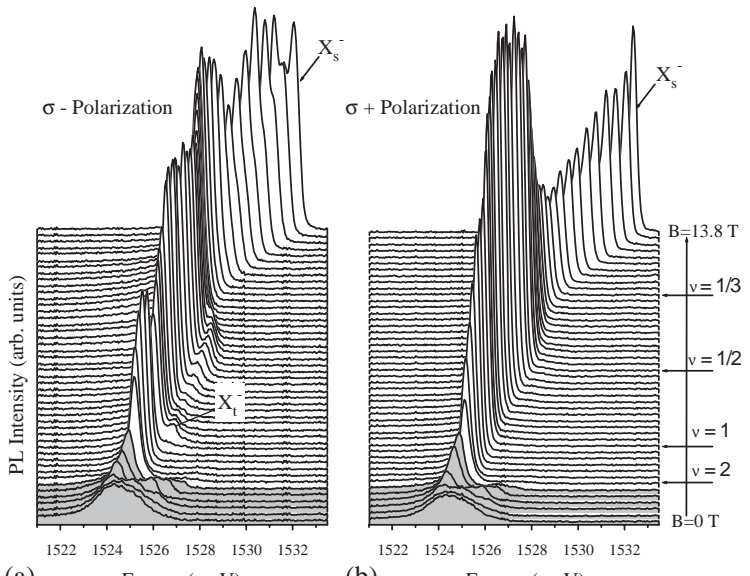

(a)

Energy (meV)

(b)

Fig. 1. PL spectra for (a) $\sigma-$, and (b) $\sigma+$ polarizations for different magnetic fields.

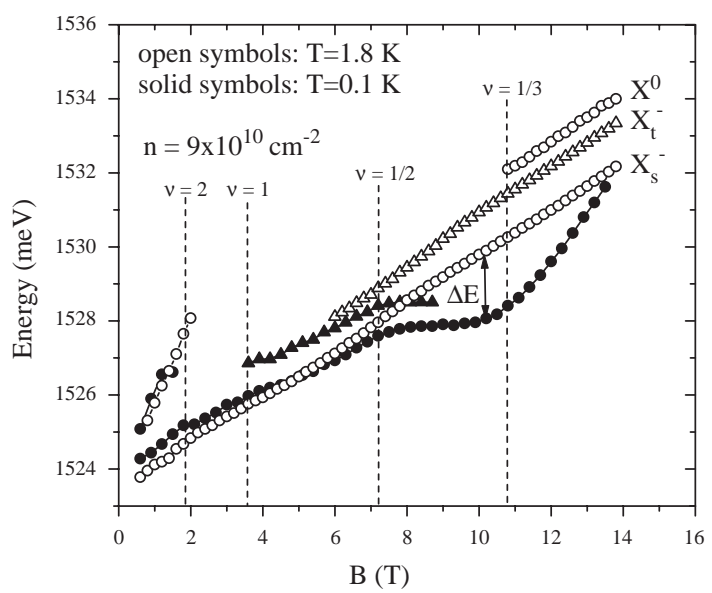

Fig. 2. Plot of the PL mode positions in $\sigma-$ polarization versus magnetic field for $T=0.1 \mathrm{~K}$ (solid symbols) and $T=1.3 \mathrm{~K}$ (open symbols).

for $v<2$ the well known charged excitons, the singlet exciton $\mathrm{X}_{\mathrm{S}}^{-}$(in both polarizations) and the triplet exciton $X_{t}^{-}$(in $\sigma$ - polarization), appear. Such a crossover from a 2DES to an excitonic system at $v=2$ is well established in the literature [14]. However, around filling factor $v=\frac{1}{3}$, a relatively strong downshift in energy of the $\mathrm{X}_{\mathrm{s}}^{-}$is observed. This can be more clearly seen in Fig. 2 where the magnetic field dispersions of the observed modes is shown (solid symbols). The open symbols in Fig. 2 mark the positions observed on the same sample at $T=1.3 \mathrm{~K}$. Obviously, there the 
anomaly is not present, rather the usual charged excitons, and, at very high fields, the neutral excitons $\left(\mathrm{X}^{0}\right)$ were observed. The most intriguing puzzle in this experiment is that a thermal energy of about $0.2 \mathrm{meV}$ is sufficient to destroy an effect which is about $2 \mathrm{meV}$ (labeled as $\Delta E$ in Fig. 2). This rules out any trivial localization effect of excitons [13]. It is clear that the anomaly is closely related to the FQHE states. We note that also transmission experiments [13] show the same anomaly. From these results one can infer that the anomaly is an intrinsic effect and is not caused by localization of excitons.

Some important facts related to the anomaly are: (a) Because of the low mobility and relatively low density of the sample, excitons are expected to remain localized. (b) The anomaly appears near $\frac{1}{3}$ i.e., excitons are near an incompressible liquid. (c) The most intriguing observation is that a very small thermal energy $(\ll 2 \mathrm{meV})$ is required to destroy the anomaly. (d) The anomaly does not appear near $v=1,2$ and is therefore an indication that the lowest-energy charged excitations at $v=\frac{1}{3}$, the quasiholes are perhaps involved in the process. The quasielectrons are predicted to have higher energies [3].

In our explanation of the observed anomaly we assume that, as a result of potential fluctuations due to impurities in the system, excitons remain localized but they are in close proximity to the incompressible liquid at $v=\frac{1}{3}$. Two of us recently investigated a system [16] where a parabolic quantum dot (QD) [15] is coupled (via the Coulomb force) to a 2DES which is in a $v=\frac{1}{3}$ Laughlin state. Electrons in the dot are confined by a parabolic potential [15], $V_{\text {conf }}(x, y)=\frac{1}{2} m^{*} \omega_{0}^{2}\left(x^{2}+y^{2}\right)$, where $\omega_{0}$ is the confinement potential strength and the corresponding oscillator length is $l_{\text {dot }}=\left(\hbar / m^{*} \omega_{0}\right)^{1 / 2}$. Calculating the low-energy excitations of that quantum dot-liquid system (a qd-liquid) we found that for a single electron in the dot the physics is somewhat similar to that of a point impurity in a $v=\frac{1}{3}$ liquid state investigated earlier [17]. In this case, the QD emits a fractionally charged quasihole $(e / 3)$ that orbits around the QD, as evidenced from the charge-density calculations $[16,17]$. Here we propose that the observed anomaly is related to the qd-liquid where the QD contains a charged exciton. The QD in our model of Ref. [16] represents a localized exciton (charged or neutral) in the present case, and perturbs the incompressible fluid due to its close proximity by creating

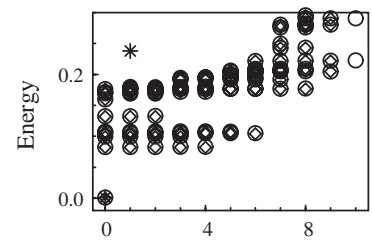

(a)

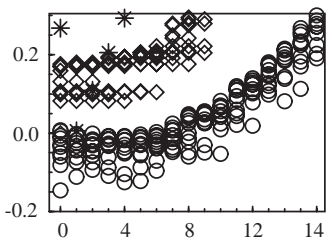

(b) Angular Momentum M

Fig. 3. Energy (in units of Coulomb energy) versus the azimuthal rotational quantum number $M$ for an isolated quantum dot $(*)$, a two-dimensional electron liquid $(\diamond)$, and a qd-liquid $(\circ)$. The QD of the qd-liquid either contains $(1 e, h)$ [in (a)], or $(2 e, h)$ [in (b)].

fractionally charged defects. Details on the formal aspects of our theory can be found in Ref. [16]. We model the incompressible state at $v=\frac{1}{3}$ filling using the spherical geometry [6] for six electrons. Electrons are treated as spinless particles corresponding to the state described by the Laughlin wave function [1]. We consider the QD size $l_{\mathrm{dot}}=15 \mathrm{~nm}$ and the liquid-dot separation $d=1.5 l_{0}$. The QD contains either a pair of electron and hole (e,h) (charge-neutral QD), or (2e,h) (charged QD). Fig. 3 shows the energy spectra for the qd-liquid where the QD contains either (e,h) [in (a)] or $(2 \mathrm{e}, \mathrm{h})$ [in (b)]. In the figures, the energy spectra of isolated dots $(*)$, an incompressible liquid at $v=\frac{1}{3}$ state $(\diamond)$ and the binding energy of the QD to the incompressible liquid ( $\mathrm{O}$ ) are plotted for comparison.

From Fig. 3(a) we can see that for a charge-neutral dot there is no dispersion of the energy as a function of $M$, and most importantly, the incompressible liquid is not influenced by the dot at all. On the other hand, the energy of the qd-liquid is significantly lowered for a charged QD, as compared to the isolated QD or the incompressible liquid without the dot [Fig. 3(b)]. This is in line with the experimental observation where only the charged excitons show the anomaly by lowering the energy.

Fig. 4 exhibits the electron density distribution in the liquid (L) and in the dot (QD) for the lowest states and for a given angular momentum of the qd-liquid system. The electron (or hole) system in the dot is close to the ground state of an isolated dot, i.e., the influence of the incompressible liquid on it is very small. On the other hand, the low-lying excited states of the qd-liquid can be described by the process of ionization as emission of a quasihole: The position of the local minimum at different angular momenta 


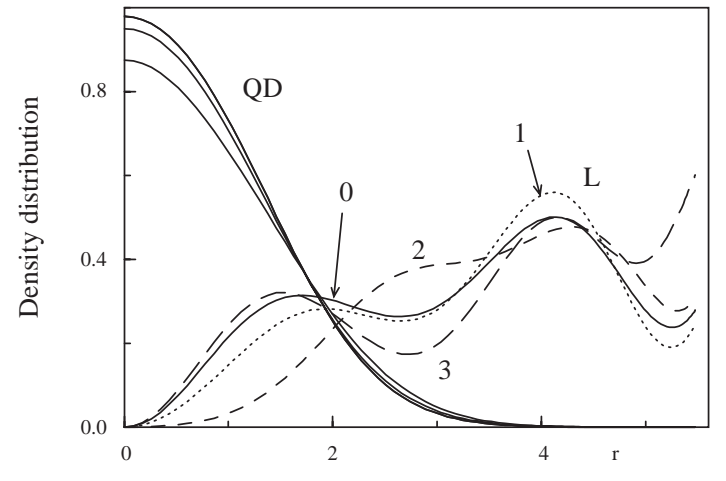

Fig. 4. Charge-density profile of the ground state and low-lying excitations of electrons in the dot (QD) and the liquid (L) of a qd-liquid at $v=\frac{1}{3}$ (for $M=0-3$, as indicated in the figure) corresponding to Fig. 3(b).

of the charge density correspond to the orbit radius of the quasihole $[17,16]$. We have evaluated the quasihole creation energy in a qd-liquid. For the Laughlin state in a pure 2DES it is $0.0276 e^{2} / \varepsilon l_{0}$ [4]. For the qd-liquid, the corresponding value is $0.32 \mathrm{meV}$ and is expected to decrease a little further with increasing number of electrons in the system representing the incompressible liquid [6]. This result indicates that the small thermal energy of about $0.2 \mathrm{meV}$ required to destroy the anomaly is in fact, the quasihole energy gap.

In conclusion, PL experiments on a 2DES subjected to a quantizing magnetic field exhibit a significant lowering of exciton energies at and around $v=\frac{1}{3}$. This is explained as due to perturbation of the incompressible liquid at $v=\frac{1}{3}$ by a localized charged exciton which results in the creation of a fractionally charged quasihole in the liquid.

We would like to thank Peter Maksym (Leicester, UK) for valuable discussions on the model described here. This work was supported by the Deutsche Forschungsgemeinschaft via SFB 508 (Hamburg) and SFB 348 (Munich), projects Schu1171/1-2, We1630/3-2, and a Heisenberg grant (Schu1171/2-1). Support by the BMBF via project BMBF 01BM918 is gratefully acknowledged.

\section{References}

[1] R.B. Laughlin, Phys. Rev. Lett. 50 (1983) 1395; R.B. Laughlin, Surf. Sci. 142 (1984) 163.
[2] B.I. Halperin, Helv. Phys. Acta 56 (1983) 75.

[3] T. Chakraborty, P. Pietiläinen, The Quantum Hall Effects, 2nd Edition, Springer, Berlin, 1995;

T. Chakraborty, Adv. Phys. 49 (2000) 959;

T. Chakraborty, in: P.T. Landsberg (Ed.), Handbook on Semiconductors, Vol. 1, Elsevier, New York, 1992, Chapter 17.

[4] T. Chakraborty, Phys. Rev. B 31 (1985) 4026.

[5] G.S. Boebinger, A.M. Chang, H.L. Störmer, D.C. Tsui, Phys. Rev. Lett. 55 (1985) 1606;

R.G. Clark, S.R. Haynes, A.M. Suckling, J.R. Mallett, P.A. Wright, J.J. Harris, C.T. Foxon, Phys. Rev. Lett. 62 (1989) 1536 ;

J.P. Eisenstein, H.L. Stormer, L. Pfeiffer, K.W. West, Phys. Rev. Lett. 62 (1989) 1540.

[6] F.D.M. Haldane, Phys. Rev. Lett. 51 (1983) 605; G. Fano, F. Ortolani, E. Colombo, Phys. Rev. B 34 (1989) 2670 ;

R. Morf, B.I. Halperin, Phys. Rev. B 33 (1986) 2221.

[7] R. de-Picciotto, M. Reznikov, M. Heiblum, V. Umansky, G. Bunin, D. Mahalu, Nature (London) 389 (1997) 162;

L. Saminadayar, D.C. Glattli, Y. Jin, B. Etienne, Phys. Rev. Lett. 79 (1997) 2526.

[8] A.J. Turberfield, S.R. Haynes, P.A. Wright, R.A. Ford, R.G. Clark, J.F. Ryan, J.J. Harris, C.T. Foxon, Phys. Rev. Lett. 65 (1990) 637;

B.B. Goldberg, D. Heiman, A. Pinczuk, L. Pfeiffer, K. West, Phys. Rev. Lett. 65 (1990) 641;

L. Gravier, M. Potemski, P. Hawrylak, B. Etienne, Phys. Rev. Lett. 80 (1998) 3344;

I.V. Kukushkin, K.v. Klitzing, K. Eberl, Phys. Rev. Lett. 82 (1999) 3665;

I.V. Kukushkin, J.H. Smet, K. von Klitzing, K. Eberl, Phys. Rev. Lett. 85 (2000) 3688.

[9] G. Finkelstein, H. Shtrikman, I. Bar-Joseph, Phys. Rev. Lett. 74 (1995) 976;

A.J. Shields, J.L. Osborne, M.Y. Simmons, M. Pepper, D.A. Ritchie, Phys. Rev. B 52 (1995) R5523.

[10] G. Yusa, H. Shtrikman, I. Bar-Joseph, Phys. Rev. Lett. 87 (2001) 216402.

[11] C. Schüller, K.-B. Broocks, Ch. Heyn, D. Heitmann, Phys. Rev. B 65 (2002) 081301(R).

[12] T. Vanhoucke, M. Hayne, M. Henini, V.V. Moshchalkov, Phys. Rev. B 65 (2002) 233305.

[13] K.-B. Broocks, P. Schröter, D. Heitmann, Ch. Heyn, C. Schüller, Phys. Rev. B 66 (2002) 041309(R).

[14] D. Gekhtman, E. Cohen, A. Ron, L.N. Pfeiffer, Phys. Rev. B 54 (1996) 10320;

E.I. Rashba, M.D. Sturge, Phys. Rev. B 54 (2000) 045305.

[15] P.A. Maksym, T. Chakraborty, Phys. Rev. Lett. 65 (1990) 108 ;

T. Chakraborty, Comments Condens. Matter Phys. 16 (1992) 35 ;

T. Chakraborty, Quantum Dots, North-Holland, Amsterdam, 1999.

[16] V.M. Apalkov, T. Chakraborty, Physica E 14 (2002) 289.

[17] E.H. Rezayi, F.D.M. Haldane, Phys. Rev. B 32 (1985) 6924. 